\title{
Laser Lock-in Thermal Wave Imaging for Nondestructive Evaluation
}

\author{
Yun-Kyu An*, Hoon Sohn*† and Ji Min Kim*
}

\begin{abstract}
This paper presents a new laser lock-in thermography (LLT) technique for nondestructive evaluation. LLT utilizes a modulated continuous wave laser beam as a heat source to obtain high fidelity thermal wave images even at the presence of background heat disturbances. The thermal waves propagating along the surface and through-the-thickness directions of a structure are visualized using newly developed laser lock-in amplitude and phase images, enhancing the detectability of surface and subsurface defects. The LLT technique is numerically investigated and experimentally validated using thermal images obtained from a steel specimen with low emissivity.
\end{abstract}

Keywords: Laser Lock-in Thermography, Noncontact Thermal Wave Imaging, Laser Lock-in Amplitude Image, Laser Lock-in Phase Image, Nondestructive Evaluation

\section{Introduction}

Infrared (IR) thermography techniques developed for nondestructive evaluation (NDE) are gaining popularity because they are noncontact, nonintrusive, rapidly deployable and applicable under harsh environments. The IR techniques can be classified into two groups: active and passive ones. Pulse phase thermography[1-3], lock-in thermography[3,4] and frequency modulated thermography[5,6] are most widely accepted active techniques. The active techniques are often advantageous over the passive ones because they have a control over an external heat source such as a flash or halogen lamp. However, the heat intensity and positioning of the heat source on the target surface cannot be precisely controlled, and the heat transmission distance is often limited due to the divergence and attenuation of the heat source. Furthermore, these conventional active techniques generate thermal wave propagation mainly in the through-the-thickness (longitudinal) direction, although thermal waves along a surface (transverse) direction are more sensitive to a surface defect.

To overcome the limitations, laser thermography techniques are recently proposed [7,8]. When a laser beam is used as a heating source, it can be transmitted over a long distance with little energy loss and precisely focused on a specific target point. Moreover, the laser beam can generates thermal waves propagating along the transverse direction, thus enabling to detect a surface defect. On the other hand, the laser thermography is often insensitive to a subsurface defect, and its application is confined to materials with high emissivity. When a laser beam is applied to a target structure with low emissivity, the majority of the transmitted laser beam is reflected and only small fraction is absorbed by the structure, making it difficult to create thermal waves. Furthermore, a high level of heat reflection caused by surrounding random heat sources hinders an IR camera from measuring actual surface thermal waves. A special surface coating can be applied to increase the emissivity[7], but such surface treatment can be cumbersome and have limited uses. Instead, a substantially high power laser

[Received: June 20, 2013, Revised: August 6, 2013, Accepted: August 12, 2013] *Department of Civil and Environmental Engineering, KAIST, 291, Daehak-ro, Yuseong-gu, Deajeon 305-701, Korea, †Corresponding Author: hoonsohn@kaist.ac.kr 
beam can be employed to have the structure absorb more energy, but then the repeated exposure of the target surface to the high power laser may cause surface ablation[9].

To cope with the prescribed limitations of the laser thermography, we propose a new thermal wave imaging technique coined as laser lock-in thermography (LLT) in this paper. The main advantages of LLT are that (1) thermal wave propagation can be visualized even when heat disturbances emitted from other surrounding heat sources are reflected from the target surface, (2) the detectability of surface and subsurface defects can be enhanced using lock-in amplitude and phase images and (3) the surface ablation can be avoided by employing a continuous wave (CW) laser source with low peak power for thermal wave generation.

\section{Laser Lock-in Thermography}

\subsection{Principle}

The principle of LLT is as follows. The CW laser beam with a periodic square waveform as shown in Fig. 1(a) is generated and illuminated onto a target surface. Then, the target surface undergoes repeated heating and cooling processes. The laser-induced thermal wave propagation can be independently investigated for two distinctive regions: Region $\mathbf{A}$ is defined as the area where transverse wave propagation can be ignored within the zone directly exposed to the excitation laser beam, and region $\mathbf{B}$ is outside region $\mathbf{A}$. Within region $\mathbf{A}, T_{I}^{A}$ propagating in the longitudinal direction is dominant, and transverse wave propagation can be ignored because longitudinal thermal gradient is much larger than transverse gradient. During the heating process, $T_{I}^{A}$ is prevailing while only $T_{R}^{A}$ exists during the cooling process as depicted in Fig. 1(b). In region $\mathbf{B}, T_{I}^{B}$ propagates in the transverse and diagonal directions during the heating process, and $T_{I}^{B}$ and $T_{R}^{B}$ coexist during the cooling process.

A 2D FE analysis of the thermal wave propagation on a mild steel plate is performed to obtain typical laser-induced thermal responses on the surface. The mild steel plate has thermal conductivity of $40 \mathrm{~W} / \mathrm{m} \cdot \mathrm{K}$, mass density of $7850 \mathrm{~kg} / \mathrm{m}^{3}$ and specific heat of $500 \mathrm{~J} / \mathrm{kg} \cdot \mathrm{K}$. The laser beam diameter is $10 \mathrm{~mm}$, and the laser beam intensity is modulated with a period of $\mathrm{T}=2$ second. Then, the thermal responses are captured at a sampling frequency of $50 \mathrm{~Hz}$. Note that the heat convection condition between the surface of the plate model and air is adopted in the simulation.

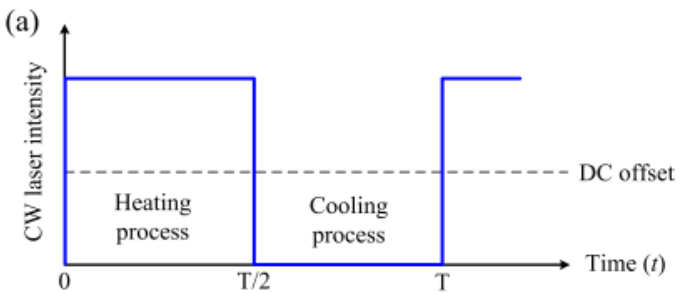

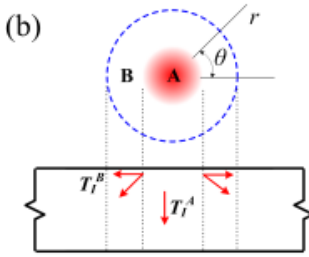

Heating process

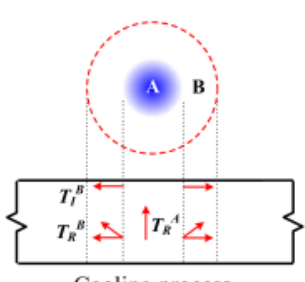

Cooling process
Fig. 1 The principle of laser lock-in thermography: (a) A modulated CW excitation laser beam with a periodic square waveform. $T$ indicates the period of the entire heating and cooling processes; (b) Laser-induced thermal wave propagation during the heating and cooling processes. "A" indicates the region where transverse wave propagation can be ignored due to direct exposure to the excitation laser beam, and "B" denotes the region outside region $A$. $T_{I}^{A}$ and $T_{R}{ }^{A}$ denote incident and reflected thermal waves within region $A$, respectively. $T_{l}^{B}$ and $T_{R}{ }^{B}$ are defined similarly. $r$ and $\theta$ are the radius and angle in the polar coordinate of the target surface. 
The representative $\mathrm{R}^{A}(t)$ and $\mathrm{R}^{B}(t)$ are displayed in Fig. 2. Fig. 2(a) shows that $\mathrm{R}^{A}(t)$ is spatially invariant inside region $\mathbf{A}$. In terms of time variation, the initial thermal value increases rapidly and then gradually approaches to its maximum at the end of the heating process. Once the heating process is transitioned to the cooling process, the thermal response again quickly drops at the initial stage and then gradually converges to its minimum value. The instant cooling results from the facts that the thermal waves on the surface quickly travel to the structural body and some of the thermal waves dissipate due to the rapid heat convection on the top surface. Then, the gradual convergence comes from $T_{R}^{A}$ traveling back to the top surface after dissipating the residual $T_{I}^{A}$ on the top surface.

On the other hand, Fig. 2(b) reveals that the peak amplitude of $\mathrm{R}^{B}(t)$ is time-delayed and attenuated as the measurement point moves further away from the epicenter of the laser spot. These phenomena are attributed to the facts that the transversely traveling $T_{I}^{B}$ and diagonally propagating $T_{R}^{B}$ need to travel a longer distance as the measurement point is shifted away from the epicenter of the laser spot.
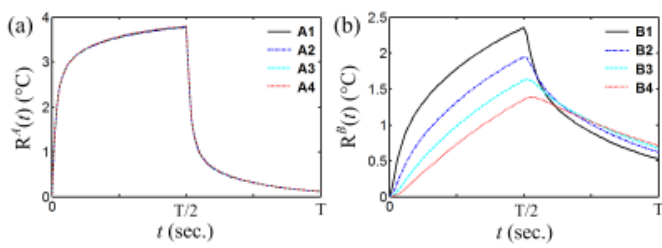

Fig. 2 The spatial and temporal variations of the representative $R(t)$ obtained from a $2 D$ FE simulation: (a) $\mathrm{R}^{A}(t)$ and (b) $\mathrm{R}^{B}(t)$ defined as $R(r, \theta, t)$ in regions $A$ and $B$, respectively. $\mathrm{R}(r, \theta, t)$ is laser-induced thermal responses, and the origin of the spatial coordinate is the epicenter of the laser source on the surface. The locations are defined as: $\mathbf{A} 1=(0,0, t), \quad \mathbf{A} 2=(0.5,0, t), \quad \mathbf{A} 3=(1,0, t)$ and $\mathbf{A} 4=$ $(1.5,0, t)$. Then, $\mathbf{B} 1=(6,0, t), \quad \mathbf{B} 2=(6.5,0, t), \quad \mathbf{B} 3=$ $(7,0, t)$ and $\mathbf{B} 4=(7 \cdot 5,0, t)$. Note that the laser beam radius is $5 \mathrm{~mm}$, and $r$ is in $\mathrm{mm}$.
Once $\mathrm{R}(r, \theta, t)$ is obtained by an IR camera on the target surface, the lock-in amplitude at a specific spatial point is defined as:

$$
A(t)=\sqrt{\{\mathrm{R}(0)-\mathrm{R}(t / 2)\}^{2}+\{\mathrm{R}(0)-\mathrm{R}(t)\}^{2}}
$$

where $0<\mathrm{t} \leq \mathrm{T} / 2$. Then, the lock-in phase at a specific spatial point is computed as:

$$
\phi(t)=\arctan [\{\mathrm{R}(0)-\mathrm{R}(\mathrm{T} / 2)\} /\{\mathrm{R}(0)-\mathrm{R}(t)\}]
$$

where $\mathrm{T} / 2<\mathrm{t} \leq \mathrm{T}$. Here, the spatial coordinate $(r, \theta)$ is omitted from both $A(t)$ and $\phi(t)$ for simplicity.

\subsection{Physical Interpretation}

To offer the physical interpretations of $A(t)$ and $\phi(t)$, their spatial and temporal variations are shown in Fig. 3.

(1) Lock-in amplitude: Fig. 3(a) shows that $A^{A}(t)$ has a pattern similar to that of $\mathrm{R}^{A}(t)$ during the heating process. The similarity between $A^{B}(t)$ and $\mathrm{R}^{B}(t)$ is also observed in Fig. 3 (b). The similarities substantiate that the lock-in amplitude physically represents the accumulation of the incident thermal wave as a function of $t$ up to the end of the
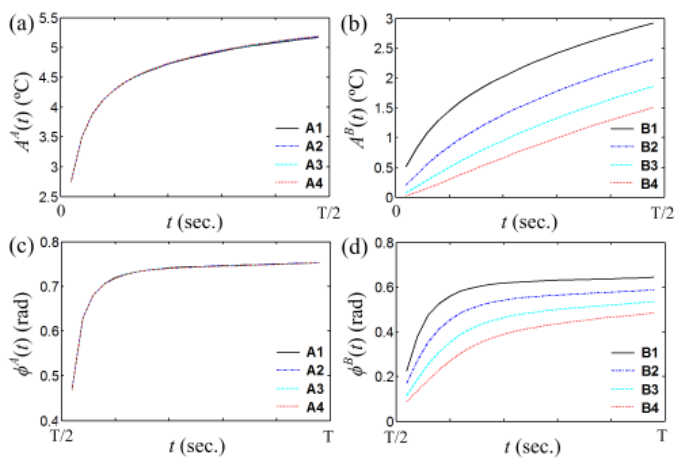

Fig. 3 The spatial and temporal variations of (a) $A^{A}(t)$, (b) $A^{B}(t)$, (c) $\phi^{A}(t)$ and (d) $\phi^{B}(t): A^{A}(t)$ and $\phi^{\mathrm{A}}(t)$ denote $A(t)$ and $\phi(t)$ within region A, respectively. $A^{B}(t)$ and $\phi^{B}(t)$ are defined similarly. 
heating process. That is, $A^{A}(t)$ shows a temperature increase purely produced by TIA, and $A^{B}(t)$ by $T_{I}^{B}$. Note that, $A^{B}(t)$ mainly captures $T_{I}^{B}$ propagating in the transverse direction and effectively visualizes transverse thermal wave propagation along the surface, making it suitable for surface defect detection in region $\mathbf{B}$. The distinct advantage of $A(t)$ over $R(t)$ is that $A(t)$ eliminates time-constant or slow-varying background noises in $R(t)$ because $A(t)$ represents only thermal variation within 0 and $t$. This attractive feature is demonstrated in Fig. 5(b).

(2) Lock-in phase: Fig. 3(c) reveals that $\phi^{A}(t)$ rapidly upsurges at the beginning of the cooling process and then gradually converges to its maximum value as $t$ increases. Because $T_{I}^{A}$ is absent during the cooling process, $\phi^{A}(t) \quad$ represents the pure longitudinal propagation of $T_{R}^{A}$, enabling subsurface defect detection within region $\mathbf{A}$. In region $\mathbf{B}, \phi^{\mathrm{B} 1}(t)$ has a steeper slope at the initial cooling stage and converges a larger value at the end of the cooling process than $\phi^{\mathrm{B} 4}(t)$ as shown in Fig. 3(d). First, the initial steeper slope pattern arises from that $T_{I}^{B}$ is delayed and attenuated as the travel distance increases. Second, the different converged values are caused by attenuation of $T_{I}^{B}$ and $T_{R}^{B}$ depending on traveling distances. Third, the converged trend is explained by the facts that $T_{R}^{B}$ is subsequently reached to the top surface after $T_{I}^{B}$ disappears. Thus, $\phi^{\mathrm{B}}(t)$ can be also used for subsurface defect detection after $T_{I}^{B}$ disappears in region B. Similar to $A(t), \phi(t)$ also removes background noises as observed in Fig. 5(c).

Once $A(\mathrm{t})$ and $\phi(t)$ values are computed for all spatial points of interest, lock-in amplitude and phase images can be constructed as a function of $\mathrm{t}$. With $A^{B}(t)$ and $\phi(t)$ images, the surface defect in region $\mathbf{B}$ and the subsurface defect in regions $\mathbf{A}$ and $\mathbf{B}$ can be effectively detected by visualizing the spatial contrasts between intact and defect areas. Note that the averaging of $\mathrm{R}(r, \theta, t)$ over the repeated heating and cooling processes improves a signal-to-noise ratio.

\section{Experimental Investigation}

\subsection{Experimental Setup}

The effectiveness of the proposed LLT technique for a laser-induced thermal wave imaging is experimentally examined. To achieve this, a LLT system is built in as shown in Fig. 4. The LLT system is composed of a laser unit, an IR camera and a personal computer (PC). The laser unit consists of an arbitrary waveform generator (AWG), a laser diode driver (LDD), a $\mathrm{CW}$ laser and a collimator. The $\mathrm{CW}$ laser used in this test has a wavelength of $808 \mathrm{~nm}$. The IR camera has a temperature resolution of $0.03{ }^{\circ} \mathrm{C}, 640 \times 480$ pixels and a wavelength of $7.5 \mu \mathrm{m}$ to $14 \mu \mathrm{m}$. The laser unit and the IR camera are controlled and synchronized using $\mathrm{PC}$, and measured thermal responses are stored and processed using MATLAB $^{\circledR}$ program installed on PC.

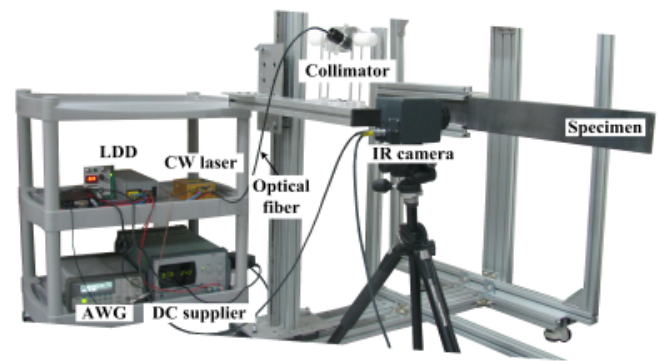

Fig. 4 Laser lock-in thermography system with a target specimen 
The test procedure is as follows. AWG is used to generate periodic square signals with a frequency of $50 \mathrm{mHz}$. The modulated voltage signal is amplified and converted into a current signal using LDD. The modulated $\mathrm{CW}$ laser beam is transmitted through an optical fiber and a collimator toward a target surface. The peak power of the $\mathrm{CW}$ laser is $3.8 \mathrm{~W}$, and the emitted beam diameter is $15 \mathrm{~mm}$. The distance between the collimator and the specimen is $600 \mathrm{~mm}$, and the IR camera is $500 \mathrm{~mm}$ apart from the specimen. The sampling rate of the IR camera is $50 \mathrm{~Hz}$. The target steel specimen has a dimension of $1000 \times 98 \times 3 \mathrm{~mm}^{3}$ and emissivity of 0.65 . Note that the emissivity is experimentally estimated using a black-body radiator.

\subsection{Experimental Results}

The representative snapshots of the raw thermal images at 5, 10, 15 and 20 seconds are shown in Fig. 5(a). Here, $\mathrm{T}=20$ second. The heat emitted from the IR camera is reflected

(a)
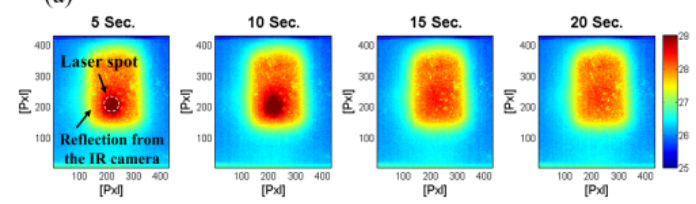

(b)
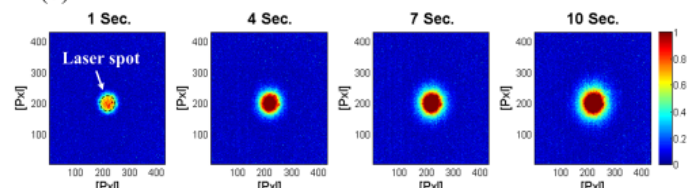

(c)
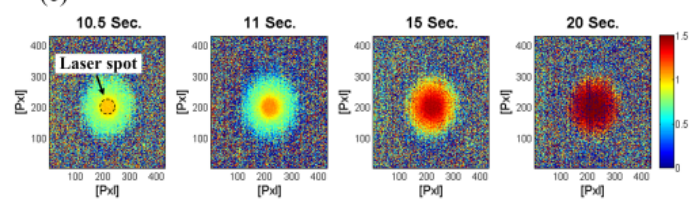

Fig. 5 Representative thermal wave propagation images: (a) Raw thermal, (b) Lock-in amplitude and (c) Lock-in phase images (Color in online) from the specimen and captured by the IR camera, creating the rectangular thermal pattern throughout the whole snapshots. Overwhelmed by this IR camera heat, laser-induced thermal waves cannot be observed properly.

In the lock-in amplitude images obtained from $t=1,4,7$ and 10 seconds, the IR camera pattern is completely removed and only the laser-induced thermal waves are clearly shown in Fig. 5(b). In region $\mathbf{B}, A^{B}(t)$ gradually increases and grows outward, showing $T_{I}^{B}$ propagation along the surface during the heating process as expected in Fig. 3(b). Similarly, the IR camera pattern is entirely eliminated from the lock-in phase images in Fig. 5(c). To capture rapid increment of $\phi^{A}(t)$ at the initial stage of the cooling process, the snapshots are taken at 10.5, 11, 15 and 20 seconds in Eq. (2). It can be observed that $\phi^{A}(t)$ rapidly increases and gradually converges to its maximum value, visualizing the longitudinal propagation of $T_{R}^{A}$. On the other hand, $\phi^{B}(t)$ increases outward from the laser center initially due to the surface traveling $T_{I}^{B}$ and later mainly due to upward diagonally propagating $T_{R}^{B}$. The test results have good agreements with theoretical and numerical expectations.

\section{Conclusion}

In this paper, a new laser thermography named laser lock-in thermography (LLT) is proposed for nondestructive evaluation (NDE) and its working principle and advantages are demonstrated. LLT makes it possible to visualize laser-induced thermal waves even at the presence of other unwanted surrounding heat sources and without any special surface treatment. Furthermore, potential surface ablation can be avoided thanks to the use of a CW laser with lower peak power. Then, LLT visualizes transverse and longitudinal thermal wave 
propagations using lock-in amplitude and phase images with the possibility of enhancing the detectability of surface and subsurface defects, respectively. Although thermal wave propagation images obtained by a single point laser excitation are demonstrated in this paper, NDE for a large structure can be achieved via laser source scanning.

\section{Acknowledgment}

This work is supported by the Technology Innovation Program (10041918) of Korea Ministry of Knowledge Economy. Any opinions, findings, and conclusions or recommendations expressed in this material are those of the author(s) and do not necessarily reflect the views of the funding agency.

\section{References}

[1] X. V. Maldague and S. Marinetti, "Pulse phase infrared thermography," Journal of Applied Physics, Vol. 79, No. 5, pp. 2697-2698 (1996)

[2] X. V. Maldague, F. Galmiche and A. Ziadi, "Advances in pulsed phase thermography," Infrared Physics and Technology, Vol. 43, No. 3-5, pp. 175-181 (2002)

[3] K. Chatterjee, S. Tuli, S. G. Pickering and D. P. Almond, "A comparison of the pulsed, lock-in and frequency modulated thermography nondestructive evaluation techniques," NDT \& E International, Vol. 44, No. 7, pp. 655-667 (2011)

[4] G. Busse, D. Wu and W. Karpen, "Thermal wave imaging with phase sensitive modulated thermography," Journal of Applied Physics, Vol. 71, No. 8, pp. 3962-3965 (1992)

[5] R. Mulaveesala and S. Tuli, "Theory of frequency modulated thermal wave imaging for nondestructive subsurface defect detection," Applied Physics Letters, Vol. 89, No. 19, 191913 (2006)

[6] V. S. Ghali, R. Mulaveesala and M. Takei, "Frequency-modulated thermal wave imaging for non-destructive testing of carbon fiber-reinforced plastic materials," Measurement Science and Technology, Vol. 22, No. 10, 104018 (2011)

[7] T. Li, D.P.Almond, D. Andrew and S. Rees, "Crack imaging by scanning pulsed laser spot thermography," NDT \& E International, Vol. 44, No. 2, pp. 216-225 (2011)

[8] J. Schlichting, C. Maierhofer and M. Kreutzbruck, "Crack sizing by laser excited thermography," NDT \& E International, Vol. 45, No. 1, pp. 133-140 (2012)

[9] C. B. Scruby and L. E. Drain, "Laser Ultrasonics: Techniques and Applications," Taylor \& Francis, London, pp. 242-249 (1990) 